fetal valproate exposure was shown to significantly impair cognitive function [2], and verbal and nonverbal abilities [3]. At the completion of the study, six-year cognitive outcome of valproate fetal-exposed children continued to exhibit significantly lower IQ than those exposed to other AEDs examined [4].

Adverse outcomes in children born to mothers with epilepsy may be caused by AEDs, maternal epilepsy, socioeconomic or genetic factors, maternal cigarette smoking, alcohol consumption, folate deficiency, or may occur by chance. Even after adjustments for these potential confounders, a follow-up study of a Danish National Birth Cohort found that preschool children exposed prenatally to AEDs had a behavioral disorder [5]; 133 children (age 4-5 years) whose mothers had received AEDs were compared to 304 unexposed and 1193 whose mothers did not have epilepsy.

\title{
References
}

1. Cohen MJ et al. Epilepsy Behav. 2013 Nov;29(2):308-15.

2. Meador KJ, et al. N Engl J Med. 2009 Apr 16;360(16):1597-605.

3. Meador KJ, et al. Brain. 2011 Feb;134(Pt 2):396-404.

4. Meador KJ, et al. Lancet Neurol. 2013 Mar;12(3):244-52.

5. Kjaer D, et al. Epilepsy Behav. 2013 Nov;29(2):407-11.

\section{EPILEPSY AND FINE MOTOR FUNCTION}

Investigators at Kocaeli University, Pediatric Neurology OP Clinic, Turkey, studied the relationship between fine motor skills and seizure and treatment parameters in 44 children with rolandic epilepsy (RE) and compared to 44 healthy controls. The children were aged 8 to 14 years, mean age 10 years, 64\% males and 36\% females, matched in age, gender and level of education in each group. WISC-R total scores were normal in both groups but the mean score in the RE group was lower than controls $(p<0.006)$. Fine motor skills as measured by the Purdue Pegboard Test (PPT) were lower in the RE group than in controls. Epileptic focus, treatment, type of treatment, age at onset of seizures, time since last seizure, and total number of seizures did not affect motor skills. RE negatively affected fine motor skills regardless of level of IQ. (Ayaz M, Kara B, Soylu N, Ayaz AB. Fine motor skills in children with rolandic epilepsy. Epilepsy Behav 2013 Nov;29(2):322-5).

COMMENTARY. Although RE is regarded as a benign disorder, several reports emphasize development of cognitive, behavioral and psychiatric disorders during the active seizure phase, sometimes persisting after the epilepsy remits [1]. Early seizures in $\mathrm{RE}$ may interfere with brain development, causing deficits in executive function despite a normal IQ. Cognitive and behavioral abnormalities may outlast the RE [2].

Month to month fluctuations in cognitive abilities and the frequency and lateralization of interictal EEG spikes are reported, potentially impacting academic performance [3]. Clinical seizure remission was achieved 4-5 years earlier than the recovery of cognitive function. No significant correlations were found between lateralization of EEG changes and the character of the cognitive dysfunction, although age-related lateralization of focal epileptiform activity was present [4]. One study suggests that valproate, ethosuximide or levetiracetam is effective in treatment of transitory cognitive disorders [4] whereas one other found that medication and duration 
of epilepsy had no significant effect on language reorganization and performance [5]. RE is associated with negative effects on fine motor skills as well as negative effects on other cognitive functions, although these may be transitory.

\section{References}

1. Neri ML, Guimaraes CA, Oliveira EP, et al. Epilepsy Behav. 2012 Aug;24(4):403-7.

2. Kwon S, Seo HE, Hwang SK. Korean J Pediatr. 2012 Oct;55(10):383-7.

3. Ewen JB, Vining EP, Smith CA, et al. Epilepsy Res. 2011 Nov;97(1-2):214-9.

4. Ermolenko NA, et al. Zh Nevrol Psikhiatr Im S S Korsakova. 2011;111(10 Pt 2):63-7.

5. Datta AN, Oser N, Bauder F, et al. Epilepsia. 2013 Mar;54(3):487-94.

\section{HYPOXIC-ISCHEMIC ENCEPHALOPATHY AND INFANTILE SPASMS}

Investigators at UCSF, British Columbia, and Toronto studied the pattern and areas of brain injury on MRI at third day of life of children with infantile spasms following neonatal hypoxic-ischemic encephalopathy (H-IE). Among a cohort of 176 term newborns with H-IE, $8(4.5 \%)$ developed infantile spasms (hypsarrhythmia in 5 or modified hypsarrhythmia in 3). Neonatal seizures occurred in $6(75 \%)$ patients who developed infantile spasms and in $9(56.3 \%)$ controls that did not. Patients with infantile spasms showed no significant differences in perinatal and neonatal course when compared with 16 controls without spasms. Development of infantile spasms after neonatal H-IE was significantly associated with basal ganglia/thalamus and total brain injury $(p=0.001)$, extent of cortical injury greater than $50 \%(p=0.01)$, injury to the midbrain $(p=0.007)$ and hypothalamic abnormalities $(p=0.01)$. Infantile spasms were significantly associated with medically refractory epilepsy and moderate to severe developmental delay. Spasms were diagnosed at a median age of 3.5 months (range 2-9 months); and at 3 months of age or younger in half of the infantile spasm patients. (Gano D, Sargent MA, Miller SP, et al. MRI findings in infants with infantile spasms after neonatal hypoxic-ischemic-encephalopathy. Pediatr Neurol 2013 Dec;49(6):401-5).

COMMENTARY. Neonatal H-IE is a relatively common precursor of infantile spasms and hypsarrhythmia. Several studies have shown that the earlier the diagnosis of infantile spasms and treatment with $\mathrm{ACTH}$, the better the response and prognosis [1][2]. The MRI is of value in the early diagnosis of infantile spasms following H-IE. Injury to the basal ganglia/thalamus and total brain injury on the third day of life are significantly associated with infantile spasms and outcome, particularly in patients with extensive cortical injury and/or injury to the midbrain. Injury to the pons or medulla is not associated with development of infantile spasms [3].

The predictive value of the EEG during early infancy for later development of West syndrome in premature infants with cystic periventricular leukomalacia (PVL) is demonstrated in a study of 19 infants with EEGs recorded at 3 months of corrected age [4]. Paroxysmal discharges during early infancy are correlated with later development of West syndrome. Prolonged EEG depression at $>21$ days of age in term and near-term infants with H-IE is a predictor of the later development of West syndrome [5].

In an ongoing investigation at Lurie Children's Hospital, Chicago, the predictive value of a pre-hypsarrhythmia pattern in serial EEGs during the first 6 months following 